\title{
Methanol masers and millimetre lines: a common origin in protostellar envelopes
}

\author{
Karl J. E. Torstensson ${ }^{1,2}$, Huib Jan van Langevelde ${ }^{1,2}$, \\ Floris F. S. van der Tak ${ }^{3,4}$, Wouter H. T. Vlemmings ${ }^{5}$, \\ Lars E. Kristensen ${ }^{2}$, Stephen Bourke ${ }^{1}$ and Anna Bartkiewicz ${ }^{6}$ \\ ${ }^{1}$ Joint Institute of VLBI in Europe, \\ PO Box 2, NL-7990 AA Dwingeloo, The Netherlands \\ email: langevelde@jive.nl \\ ${ }^{2}$ Leiden Observatory, Leiden University, \\ PO Box 9513, NL-2300 RA Leiden, The Netherlands \\ email: kalle@strw.leidenuniv.nl \\ ${ }^{3}$ SRON Netherlands Institute for Space Research, \\ Landleven 12, NL-9747 AD Groningen, The Netherlands \\ email: vdtak@sron.nl \\ ${ }^{4}$ Kapteyn Astronomical Institute \\ University of Groningen, The Netherlands \\ ${ }^{5}$ Chalmers University of Technology, Department of Earth and Space Science, \\ SE-412 96 Gothenburg, Sweden \\ email: wouter.vlemmings@chalmers.se \\ ${ }^{6}$ Toruń Centre for Astronomy, Nicolaus Copernicus University, \\ Gagarina 11, 87-100 Torun, Poland \\ email: annan@astro.uni.torun.pl
}

\begin{abstract}
To understand the origin of the $\mathrm{CH}_{3} \mathrm{OH}$ maser emission, we map the distribution and excitation of the thermal $\mathrm{CH}_{3} \mathrm{OH}$ emission in a sample of 14 relatively nearby $(<6 \mathrm{kpc})$ highmass star forming regions that are identified through $6.7 \mathrm{GHz}$ maser emission. The images are velocity-resolved and allow us to study the kinematics of the regions. Further, rotation diagrams are created to derive rotation temperatures and column densities of the large scale molecular gas. The effects of optical depth and subthermal excitation are studied with population diagrams. For eight of the sources in our sample the thermal $\mathrm{CH}_{3} \mathrm{OH}$ emission is compact and confined to a region $<0.4 \mathrm{pc}$ and with a central peak close $(<0.03 \mathrm{pc})$ to the position of the $\mathrm{CH}_{3} \mathrm{OH}$ maser emission. Four sources have more extended thermal $\mathrm{CH}_{3} \mathrm{OH}$ emission without a clear peak, and for the remaining two sources, the emission is too weak to map. The compact sources have linear velocity gradients along the semi-major axis of the emission of $0.3-13 \mathrm{~km} \mathrm{~s}^{-1} \mathrm{pc}^{-1}$. The rotation diagram analysis shows that in general the highest rotation temperature is found close to the maser position. The confined and centrally peaked $\mathrm{CH}_{3} \mathrm{OH}$ emission in the compact sources indicates a single source for the $\mathrm{CH}_{3} \mathrm{OH}$ gas and the velocity fields show signs of outflow in all but one of the sources. The high detection rate of the torsionally excited $v_{\mathrm{t}}=1$ line and signs of high- $K$ lines at the maser position indicate radiative pumping, though the general lack of measurable beam dilution effects may mean that the masing gas is not sampled well and originates in a very small region.
\end{abstract}

Keywords. Masers, stars: formation, ISM: jets and outflows, ISM: kinematics and dynamics, ISM: molecules, submillimeter 


\section{Introduction}

Though high-mass star formation has been extensively studied, the particulars of the early evolution is still widely debated and several theories have been proposed (Zinnecker $\&$ Yorke 2007). The currently favoured scenario is that of monolithic collapse or core accretion, a scaled up version of lower-mass star formation. In this scenario the accretion occurs via a disk with associated outflows. However, because of the short life time and rapid evolution, massive stars are few and far between. Furthermore, they form in a clustered environment in the densest part of the molecular cloud where they are heavily obscured. These conditions lead to several observational challenges, and high angular resolution observations at wavelengths that can penetrate the dense molecular clouds are therefore needed.

Since its discovery, the $6.7 \mathrm{GHz}$ methanol maser has been associated with the early stages of high-mass star formation (Menten 1991). Initial studies found the maser emission to be associated with ultra-compact (UC) HII regions (Phillips, Norris, Ellingsen, et al. 1998; Walsh, Burton, Hyland, et al. 1998). Follow-up studies have since shown that in general the maser emission is associated with an earlier evolutionary stage (Beuther, Walsh, Schilke, et al. 2002; Walsh, Macdonald, Alvey, et al. 2003). This scenario is supported by large studies in which most maser sources are associated with mm cores rather than UCHII regions. Moreover, searches for methanol maser emission towards low-mass star forming regions have shown the Class II masers to be exclusively associated with high-mass star formation (Minier, Ellingsen, Norris, et al. 2003; Xu, Li, Hachisuka, et al. 2008).

Detailed high-resolution VLBI studies of the methanol masers have led to several different classifications based on their morphology. It appears that the masers occur in outflows, disks, and possibly other physical structures. The extent of the masers appears to be between a few hundred and a couple of thousand AU, an observation common to all sources. A recent VLBI study of 30 methanol maser sources based on a blind survey found $30 \%$ of the masers to have an elliptical distribution (Bartkiewicz, Szymczak, van Langevelde, et al. 2009). A similar elliptical distribution has been observed in the nearby source Cepheus A HW2, for which we propose that the maser emission occurs in a shock interface in the equatorial region of the protostellar object (Torstensson, van Langevelde, Vlemmings, et al. 2011a). This geometry is supported by the magnetic field being aligned along the outflow axis, regulating the infall of the accreting material (Vlemmings, Surcis, Torstensson, et al. 2010).

To understand the origin of the $\mathrm{CH}_{3} \mathrm{OH}$ maser emission, we map the distribution and excitation of the thermal $\mathrm{CH}_{3} \mathrm{OH}$ emission in a sample of 14 relatively nearby $(<6 \mathrm{kpc})$ high-mass star forming regions that are identified through $6.7 \mathrm{GHz}$ maser emission.

\section{Observations and data reduction}

We have used the HARP-B instrument on the JCMT $\dagger$ to map the thermal $\mathrm{CH}_{3} \mathrm{OH}$ emission in the $J=7_{\mathrm{K}} \rightarrow 6_{\mathrm{K}}$ at $338 \mathrm{GHz}$ towards 14 high-mass star-forming regions associated with $6.7 \mathrm{GHz}$ methanol maser emission. The observations were performed in a jiggle-chop mode (harp5) to create $2^{\prime} \times 2^{\prime}$ maps with a pixel spacing of $6^{\prime \prime}$. This ensures proper Nyquist sampling of the $14^{\prime \prime}$ JCMT beam at $338 \mathrm{GHz}$. During the observations regular pointings were done on calibrators and we estimate an absolute pointing accuracy

$\dagger$ The James Clerk Maxwell Telescope is operated by the Joint Astronomy Centre on behalf of the Science and Technology Facilities Council of the United Kingdom, the Netherlands Organisation for Scientific Research, and the National Research Council of Canada. 
of $\sim 1^{\prime \prime}$. The $1 \mathrm{GHz}$ bandwidth covers a total of 25 lines of both $\mathrm{E}$ and $\mathrm{A}$ type $\mathrm{CH}_{3} \mathrm{OH}$ with upper energy levels between $65 \mathrm{~K}$ and $260 \mathrm{~K}$, see Fig. 1 for a sample spectrum. In our analysis we have adopted a main beam efficiency of 0.6 (Buckle, Hills, Smith, et al. 2009) and estimate a calibration uncertainty of $20 \%$.

The initial data inspection and re-gridding of the data was done with the Starlink package using Gaia/SPLAT after which the data were converted to GILDAS/CLASS format and the remaining data reduction and analysis was performed in CLASS. To increase the signal-to-noise of the spectra the data were smoothed to a velocity resolution of $0.87 \mathrm{~km} \mathrm{~s}^{-1}$, after which a linear baseline was fitted to the emission free regions of each spectra and subtracted. The analysis was then performed on a pixel by pixel basis in which the strongest unblended line in the spectra (-1E) was fitted with a Gaussian. The velocity of the $-1 \mathrm{E}$ line was then used to calculate the velocities of the other 24 methanol lines. Around each calculated velocity the moments were calculated within a window size of two times the line width of the fitted $-1 \mathrm{E}$ line. Due to line blending we have also used the first and second moment of the lines to help determine whether a line is real.

\section{Results}

The $\mathrm{CH}_{3} \mathrm{OH}-1 \mathrm{E}$ is the strongest unblended line in the spectra, and is used to map the integrated line flux, central velocity, and line width. To analyse the excitation of the largescale methanol gas, we have performed a rotational diagram analysis of each position, for which at least three lines are detected. Furthermore, a population diagram analysis was used at the position of the methanol maser emission. The population diagram analysis takes into account the optical depth of the lines and the source size, both of which can affect the measured line intensity (Torstensson, van der Tak, van Langevelde, et al. 2011b).

Four of the sources have extended and complex thermal $\mathrm{CH}_{3} \mathrm{OH}$ emission and are part of larger star-forming regions. In two of the sources the methanol emission was too weak to be mapped. The remaining eight sources all have compact thermal $\mathrm{CH}_{3} \mathrm{OH}$ emission and will be the focus of this paper.

The thermal $\mathrm{CH}_{3} \mathrm{OH}$ emission for the the eight compact sources is confined to a region with a major-axis $a<0.4 \mathrm{pc}$, and centrally peaked close to the maser position $(r<$ $0.03 \mathrm{pc}$ ), Fig. 2. Moreover, all but one of the eight sources have a linear velocity gradient of a few $\mathrm{km} \mathrm{s}^{-1} \mathrm{pc}^{-1}$ across the source.

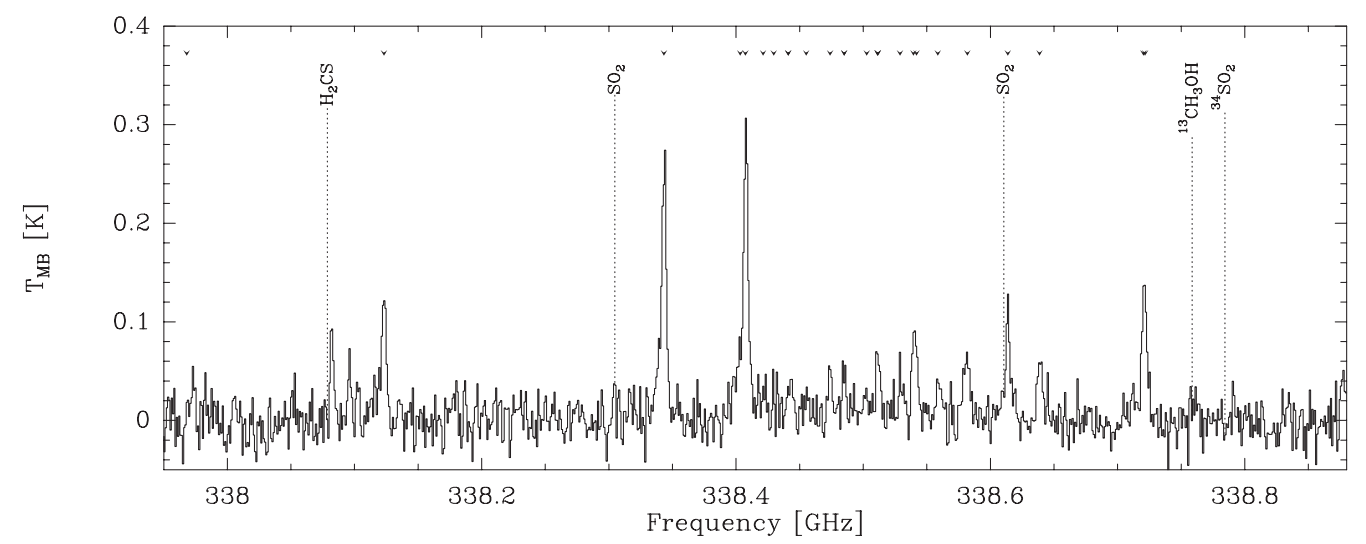

Figure 1. Sample spectrum of the source G23.389+00.185. The tick marks at the top indicate the $\mathrm{CH}_{3} \mathrm{OH} 7_{K} \rightarrow 6_{K}$ lines, also indicated are other identified species. 
The rotational analysis indicates moderate temperatures $(30-50 \mathrm{~K})$ in the more extended gas, and elevated rotation temperatures $(\sim 100 \mathrm{~K})$ at the maser position. Contrary to what is found in the source Cepheus A HW2 (Torstensson, van der Tak, van Langevelde, et al. 2011b) we only detect one single gas component in the other sources. Furthermore, despite signs of high- $K$ in some of the sources we do not find any beam dilution in the population diagram analysis and the optical depth of the low- $K$ lines is at most moderate. Still, for half of the 14 sources in our sample we detect the torsionally excited $v_{\mathrm{t}}=1$ line at the maser position.

\section{Discussion}

We have mapped the large scale distribution and excitation of the thermal methanol gas towards a sample of 14 high-mass star-forming regions associated with $6.7 \mathrm{GHz}$ methanol maser emission. Eight of the sources have compact thermal $\mathrm{CH}_{3} \mathrm{OH}$ emission with a central peak close to the maser position. The linear velocity gradient across the sources lines up with other outflows tracers and the rotational diagram analysis points to a single highly excited source at the centre. We interpret this result as the large-scale methanol gas being entrained in an outflow with a common driving source, and that it can be traced back to the protostellar object, as in the case of Cepheus A HW2 (Torstensson, van Langevelde, Vlemmings, et al. 2011a).

Because the $6.7 \mathrm{GHz}$ methanol maser emission typically has an extent of a few hundred to a couple of thousands of $\mathrm{AU}$ and is located close to the protostellar object we expect the highly excited gas associated with the maser emission to have a similar extent (Bartkiewicz, Szymczak, van Langevelde, et al. 2009). We therefore performed a population diagram analysis of the gas at the maser location to allow for source size and optical depth effects. However, the general lack of beam dilution effects that we find leads us to believe that we are not sampling the highly excited gas with these observations. Rather we are only sampling the more extended gas traced by the low- $K$ lines.

An independent indication of highly excited $\mathrm{CH}_{3} \mathrm{OH}$ gas is the detection of the $-1 \mathrm{~A}$ $v_{\mathrm{t}}=1$ line at $337.97 \mathrm{GHz}$ in half of the sources in our sample. Because collisional pumping alone is very inefficient at populating the torsionally excited state, the $v_{\mathrm{t}}=1$ line is an indicator of infrared pumping at work (Leurini, Schilke, Wyrowski, et al. 2007). However, due to the low signal-to-noise of the only torsionally excited line in our spectra we are unable constrain the radiative excitation and break the degeneracy between density and temperature. The sources for which the $v_{\mathrm{t}}=1$ line is detected also show signs of the
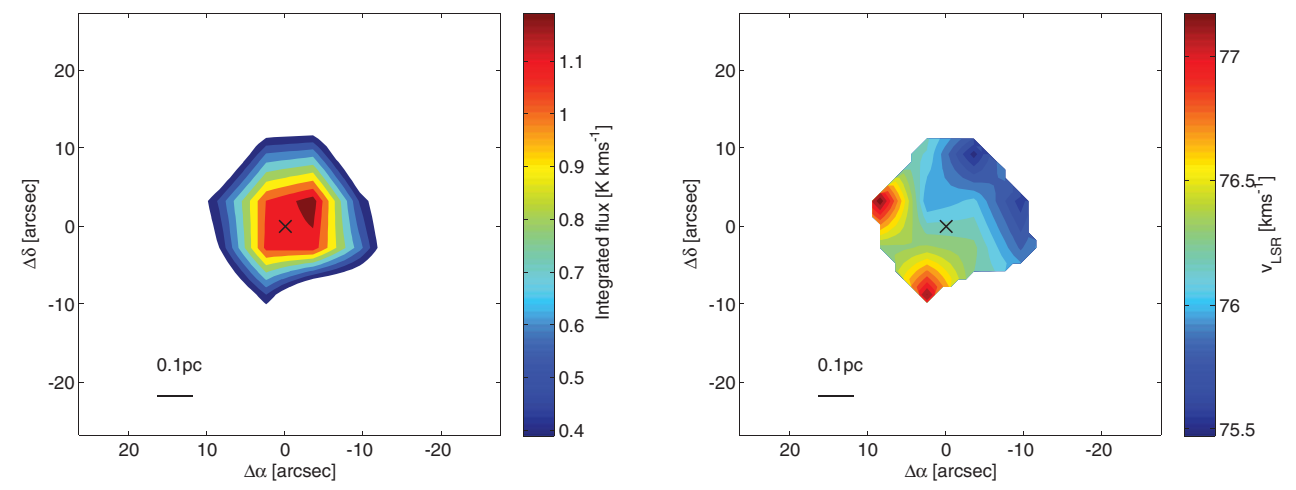

Figure 2. Integrated intensity (left) and central velocity (right) of the $\mathrm{CH}_{3} \mathrm{OH} 7_{-1} \rightarrow 6_{-1} \mathrm{E}$ line for the source G23.389+00.185. The black cross marks the methanol maser position. 
high- $K$ lines, supporting the argument of highly excited gas. Although the high- $K$ lines are weak, we have a considerable detection rate of the $v_{\mathrm{t}}=1$ line. The high detection rate of the $v_{\mathrm{t}}=1$ line could be an effect of our sample selection, because we have intentionally targeted bright $6.7 \mathrm{GHz}$ methanol maser sites, which are thought to be pumped by infrared radiation. For both the $v_{\mathrm{t}}=1$ line and the high- $K$ lines beam dilution effects are likely important. There does however not seem to be any correlation between the distance of the source or the luminosity and the occurrence of the $v_{\mathrm{t}}=1$ line which one might expect. Neither are the sources with the $v_{\mathrm{t}}=1$ line detection those with the largest integrated flux of the $-1 \mathrm{E}$ line. Instead, the $v_{\mathrm{t}}=1$ line seems to be correlated with the sources for which we find a non-negligible optical depth in the low- $K$ lines in the population diagram analysis. Higher resolution interferometric observations with beam sizes similar to the extent of the maser regions (and more accurate distance estimates) would greatly help to constrain the excitation of the highly excited gas (Beuther, Zhang, Hunter, et al. 2007).

The methanol maser emission can be described as having an elliptical distribution for three of the compact sources (Cepheus A HW2, G23.207-00.377, and G23.389+00.185) (Torstensson, van Langevelde, Vlemmings, et al. 2011a; Bartkiewicz, Szymczak, van Langevelde, et al. 2009). Assuming that the maser emission arises in the equatorial region of the protostellar object, a velocity model with a radial and a rotational velocity component can be fitted to the maser velocity field. In all three sources the radial velocity component is the dominant one. With the orientation of the larger scale thermal methanol outflow we can constrain the geometry of the systems and find that the radial velocity is that of infall rather than outflow.

All in all, our results are in agreement with the monolithic collapse scenario, in which accretion proceeds through a disk with associated outflows.

\section{References}

Bartkiewicz, A., Szymczak, M., van Langevelde, H. J., Richards, A. M. S., \& Pihlström, Y. M. 2009, A\&BA, 502, 155

Beuther, H., Walsh, A., Schilke, P., Sridharan, T. K., Menten, K. M., \& Wyrowski, F. 2002, $A \mathscr{E} A, 390,289$

Beuther, H., Zhang, Q., Hunter, T. R., Sridharan, T. K., \& Bergin, E. A. 2007, A\&A, 473, 493

Buckle, J. V., Hills, R. E., Smith, H., et al. 2009, MNRAS, 399, 1026

Leurini, S., Schilke, P., Wyrowski, F., \& Menten, K. M. 2007, A\& A, 466, 215

Menten, K. M. 1991, ApJ, 380, L75

Minier, V., Ellingsen, S. P., Norris, R. P., \& Booth, R. S. 2003, A\&A, 403, 1095

Phillips, C. J., Norris, R. P., Ellingsen, S. P., \& McCulloch, P. M 1998, MNRAS, 300, 1131

Torstensson, K. J. E., van Langevelde, H. J., Vlemmings, W. H. T., \& Bourke, S. 2011, A\&A, $526, \mathrm{~A} 38$

Torstensson, K. J. E., van der Tak, F. F. S., van Langevelde, H. J., Kristensen, L. E., \& Vlemmings, W. H. T. 2011, A\& $A, 529$, A32

Vlemmings, W. H. T., Surcis, G., Torstensson, K. J. E., \& van Langevelde, H. J. 2010, MNRAS, 404, 134

Walsh, A. J., Burton, M. G., Hyland, A. R., \& Robinson, G. 1998, MNRAS, 301, 640

Walsh, A. J., Macdonald, G. H., Alvey, N. D. S., Burton, M. G., \& Lee, J.-K. 2003, A\&A, 410, 597

Xu, Y., Li, J. J., Hachisuka, K., Pandian, J. D., Menten, K. M., \& Henkel, C. 2008, A $\mho$ A, 485, 729

Zinnecker, H. \& Yorke, H. W. 2007, ARAEA A, 45, 481 